\title{
Optimization Analysis on Layout of 220kV Outdoor Substation for Noise Control at the Boundary
}

\author{
Lu Mengyao $^{1 *}$ \\ ${ }^{1}$ College of Electrical Engineering \& New Energy, China Three Gorges Univ., Yichang 443002, China
}

\begin{abstract}
The noise in substations has become one of the most concerning problems in the field of power grid. In this paper, the principle of substation layout optimization based on the maximum acoustic ray shielding method is established in the premise of the sound wave propagation rule in complicated air medium to meet the requirements of relevant national environmental standards for noise at the boundary of the substation. By establishing the acoustic simulation analysis model of the $220 \mathrm{kV}$ outdoor substation, the noise level at the boundary of the substation before and after the layout optimization is compared and analysed, and the distribution rule of the sound field inside and outside the substation is obtained. The analysis results show that the optimized layout of the substation can effectively reduce the noise at the boundary of the substation, which provides a control method for the noise optimization design of the substation.
\end{abstract}

\section{Introduction}

With the rapid development of power grid construction and urban development, the contradiction between the environmental impact caused by substation operation and the people's growing awareness in environmental protection is increasingly prominent. The noise generated during the operation of a substation will inevitably affect the nearby residents and the environment. Due to people's increasing awareness of environmental protection and great attention to the environmental impact of power transmission and transformation projects, the noise in substation has become one of the most concerning problems in the field of power system. It has become an important prerequisite for the design and construction of power transmission and transformation project that the noise at the boundary of a substation meets the requirements of relevant national environmental standards ${ }^{[1]}$. Therefore, how to further reduce the impact of noise in substation on the surrounding environment and create a green environmental protection power transmission and transformation project has become an important problem faced by the current power grid construction. In this paper, the method of substation layout optimization based on the maximum acoustic ray shielding method is established aiming at the actual needs of noise control and based on the sound wave propagation rule in complicated air medium [2]. The acoustic simulation analysis model of a $220 \mathrm{kV}$ outdoor substation is established with numerical analysis method, and the noise level at the boundary of the substation before and after the layout optimization is compared and analysed, so as to find a scientific and effective optimization layout method for the noise control in the substation.

\section{Maximum acoustic ray shielding method}

Sound waves propagate in all directions in air or other medium. The sound becomes weaker and weaker during propagating, that is, the attenuation of sound waves. Generally, the noise at the boundary is controlled by reducing the radiation level of the sound source, blocking the sound propagation path and protecting the receiving point ${ }^{[3]}$. The method of blocking the sound propagation path is more convenient and widely used, which is mainly realized by general sound insulation measures. The noise reduction principle of sound insulation is as follows: when encountering a sound insulation barrier, the sound wave propagating in the air will develop the phenomenon of reflection, transmission and diffraction ${ }^{[4]}$. Some of the sound waves pass through the top of the sound insulation barrier and diffract to the sound receiving point, some of them pass through the sound insulation barrier and reach the receiving point, and some of them reflect on the wall of the sound insulation barrier. The insertion loss of the sound insulation barrier mainly depends on the sound energy distribution of the sound wave from the sound source along the three routes. The sound insulation barrier aims to block the transmission of direct sound, isolate the transmitted sound and bring enough attenuate to the diffraction sound ${ }^{[5]}$. 


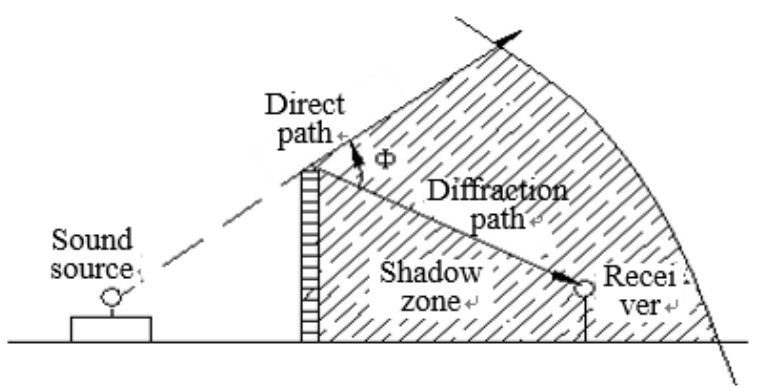

Figure 1. Diffraction of Sound Barrier

As shown in Figure 1, when the sound waves hits the wall of the sound insulation barrier, it will produce diffraction at the edge of the sound insulation barrier, thus forming a sound shadow zone behind the sound insulation barrier. The expected noise reduction effect of a sound insulation barrier expected is in the range of the sound shadow zone. Compared with the light shadow zone, the boundary of this sound shadow zone is not obvious due to the sound wavelength being further longer than the light wave wavelength. Beyond the edge of the sound insulation barrier, the range that the sound wave from the sound source can directly reach is called the "bright zone". There is a small transition zone from the bright zone to the sound shadow zone. The noise level in the sound shadow zone and transition zone is lower than that without sound insulation barrier, which is the basic principle of noise reduction of the sound insulation barrier ${ }^{[6]}$.

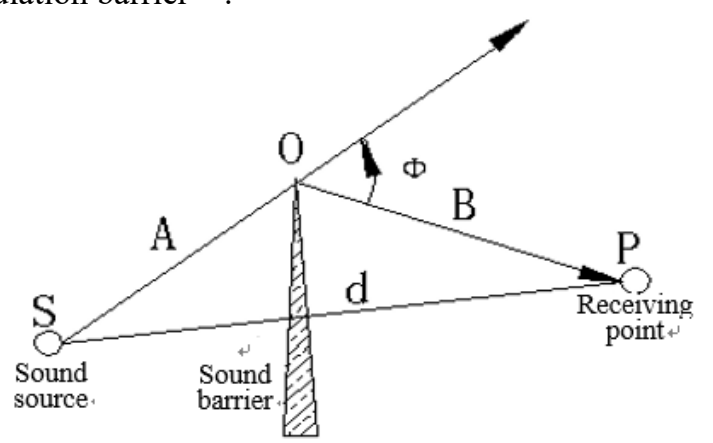

Figure 2. Sound Path Difference

As shown in Figure 2, it is assumed that an infinite sound barrier with sufficient zone density is set between the sound source and the receiver. In this case, the sound wave can only be diffracted through the top of the sound barrier to the receiver. There is a diffraction angle $\varphi$ when the sound reaches the receiver. The area of diffraction bending is the sound shadow zone, and the receiver in the sound shadow zone can feel that the noise has been significantly attenuated. The effect of noise reduction of the sound barrier depends on the diffraction angle $\varphi$. The larger the diffraction angle is, the greater the sound attenuation of the sound barrier, and the better the effect ${ }^{[7]}$. Obviously, if the height of the sound insulation barrier is increased, or the sound source or the receiver is close to the sound barrier, the diffraction angle can be increased. The attenuation effect of sound insulation barrier is a function of the geometric relationship among sound source, receiver and sound barrier. Where, the distance difference of sound waves is defined as $\delta(\delta=\mathrm{A}$ $+\mathrm{B}-\mathrm{d})$.

For the same sound barrier, the noise attenuation values of different frequencies are different at different positions in the "sound shadow zone". Due to the limited quantity of buildings, sometimes it is difficult to block the noise radiation in all directions ${ }^{[8]}$. At this time, priority can be given to the direction of the maximum acoustic ray for building layout. The so-called acoustic ray is a curve which is emitted from the sound source and represents the direction of sound energy propagation. In a variety of homogeneous medium, the acoustic ray represents the direction of wave propagation and is perpendicular to the wave front everywhere. Because of refraction and reflection, the acoustic ray is not necessarily a straight line, it also can be a polygonal line or a curve, but the acoustic ray is always orthogonal to the wave front ${ }^{[9]}$. The direction of the maximum acoustic ray refers to the direction where the noise radiates the strongest outward. The inherent buildings in the substation can effectively block the noise from the substation from radiating outward. When setting the buildings in the substation, the buildings should be arranged on the path of the maximum acoustic ray. This layout optimization method is called maximum acoustic ray shielding method. Therefore, in the design of the substation, the noise radiation from the substation can be effectively reduced by placing the main control communication building, station power room, relay room and other buildings on the path of the maximum acoustic ray of the main transformer ${ }^{[10]}$.

\section{Noise analysis of the $220 \mathrm{kV}$ outdoor substation}

The number of $220 \mathrm{kV}$ outdoor substation is large, the equipment noise is large, and the risk of noise exceeding the standard is high. Therefore, in this paper, the $220 \mathrm{kV}$ outdoor substation is selected as the analysis object. Two 180MVA transformers are installed in the substation, and the layout of station area is shown in Figure 3.

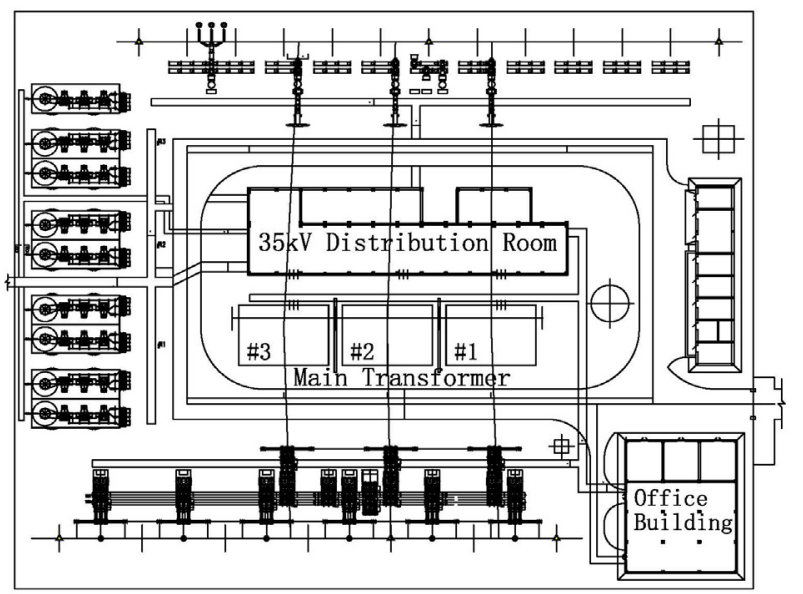

Figure 3. Layout of $220 \mathrm{kV}$ Substation

Computer noise prediction software, which is an important auxiliary tool, is often used to calculate and analyse the noise intensity inside and outside the 
boundary of the substation and noise sensitive points. It plays an important role in dealing with the noise problems inside and outside a new or existing substation. In this paper, a three-dimensional model of the $220 \mathrm{kV}$ substation is established according to the internal building and equipment distribution of the substation, as shown in Figure 4.

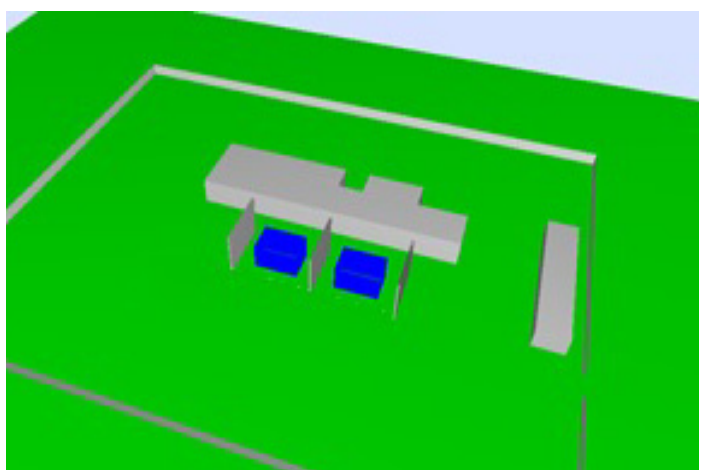

Figure 4. Three-dimensional Model of $220 \mathrm{kV}$ Substation

The enclosure is $2.3 \mathrm{~m}$ high and the firewall is $7.5 \mathrm{~m}$ high. All buildings are modelled according to the actual size. The main sound source is two main transformers. The sound pressure level adopts the actual test value and the calculated elevation is $1.5 \mathrm{~m}$. The software calculation is based on the calculation of atmospheric sound absorption and the attenuation methods of various physical mechanisms in ISO 9613:1996 Acoustics Attenuation of Sound During Propagation Outdoors Part 2: General Method of Calculation. The physical effects considered include: geometric divergence, atmospheric absorption, ground effect, surface reflection and shielding caused by obstacles. Finally, the noise distribution at the boundary of the substation is shown in Figure 5.

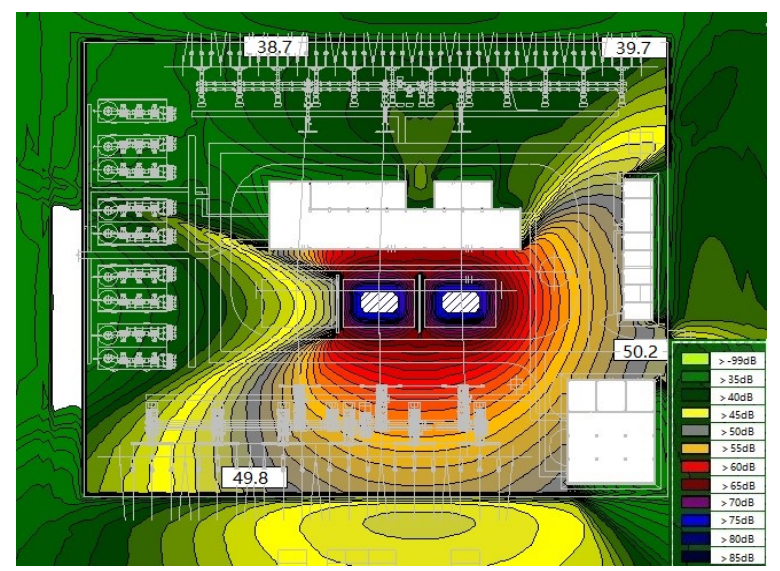

Figure 5. Noise Distribution at the Boundary of the Substation

Combined with the measured noise data of four measuring points around the boundary of the substation, the noise prediction and analysis results are compared with the measured noise values in the field, as shown in Table 1.
Table 1. Comparison of Predicted and Measured Noise Values at Measuring Points

\begin{tabular}{cccc}
\hline $\begin{array}{c}\text { Measuring } \\
\text { point }\end{array}$ & $\begin{array}{c}\text { Predicted } \\
\text { value (dB (A)) }\end{array}$ & $\begin{array}{c}\text { Measured } \\
\text { value } \\
(\mathrm{dB}(\mathrm{A}))\end{array}$ & $\begin{array}{c}\text { Difference } \\
(\mathrm{dB}(\mathrm{A}))\end{array}$ \\
\hline 1 & 50.2 & 50.4 & -0.2 \\
2 & 39.7 & 39.7 & 0.0 \\
3 & 38.7 & 38.7 & 0.0 \\
4 & 49.8 & 50.6 & -0.8 \\
\hline
\end{tabular}

It can be seen from table 1 that the error between the noise prediction result and the measured value of the $220 \mathrm{kV}$ substation with calculation and analysis software is within $1 \mathrm{~dB}(\mathrm{~A})$. Considering the influence of the production process of the capacitor at the boundary side and the absence of damping pad on the field measurement results, it can be considered that the modelling of the substation sound source and buildings is reasonable, the parameters are set correctly and the noise prediction results are accurate.

\section{Layout optimization and noise analysis of substation}

The maximum acoustic ray of the $220 \mathrm{kV}$ outdoor substation is the center line of two main transformers. Due to the shielding effect of the main control building at the north side of the main transformer on the maximum acoustic ray, the noise value of the boundary at the north side is lower; however, due to the absence of buildings from the south side of the main transformer to the boundary, the noise value of the boundary at the south side is relatively high, so the maximum acoustic ray shielding method can be adopted to change the $220 \mathrm{kV}$ GIS room in the southeast side into a strip-shaped room; the main transformer interval and outgoing line interval share a row of joint structures, and the whole strip-shaped building is moved to the south side of the main transformer, so as to control the noise at the south side of the boundary by shielding the maximum acoustic ray. In this arrangement, two fire doors will be added to the $220 \mathrm{kV}$ GIS room, but it can better solve the problem of excessive noise at the south side of the main transformer. At the same time, considering that the noise value at the northeast boundary is high, the building geometry adjustment method can be used to appropriately lengthen the vertical length between the main control building in the north and the sound source, so as to reduce the noise at the northeast boundary. Based on the above measures, a firewall can be added to the east side of the main transformer to shield the eastwest acoustic ray, so as to reduce the noise at the east side of the boundary. After adopting the above measures, the noise analysis model of the substation after optimization is shown in Figure 6, and the prediction result of noise distribution is shown in Figure 7. 


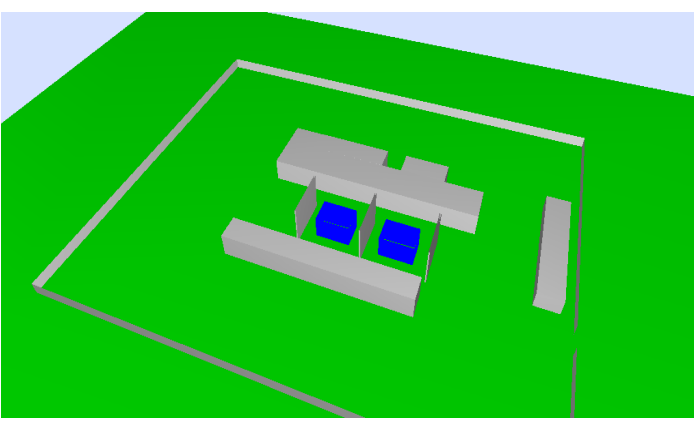

Figure 6. Layout Optimization Model of Substation

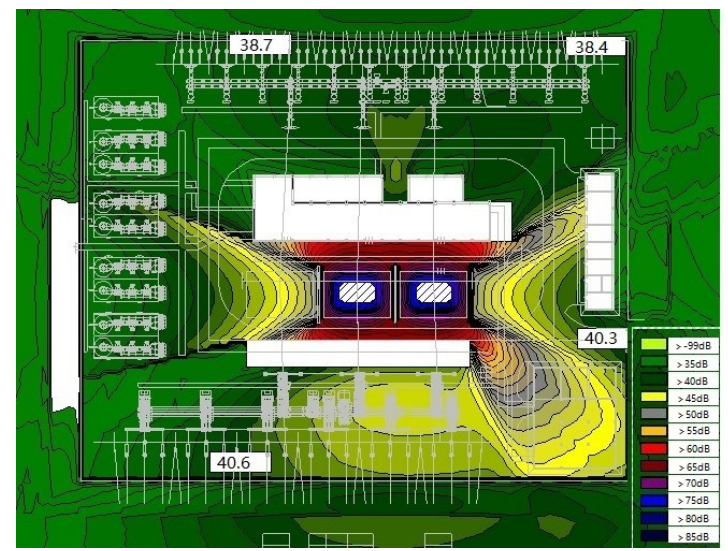

Figure 7. Noise Distribution in Optimization Model at the Boundary of the Substation

The noise comparison at the measuring points of the boundary of $220 \mathrm{kV}$ outdoor substation before and after the layout optimization design is shown in Table 2.

Table 2. Comparison of Predicted Noise Values at Measuring Points Before and After Layout Optimization

\begin{tabular}{cccc}
\hline $\begin{array}{c}\text { Measuring } \\
\text { point }\end{array}$ & $\begin{array}{c}\text { Noise value } \\
\text { after } \\
\text { optimization } \\
\mathrm{dB}(\mathrm{A})\end{array}$ & $\begin{array}{c}\text { Noise value } \\
\text { before } \\
\text { optimization } \\
\mathrm{dB}(\mathrm{A})\end{array}$ & $\begin{array}{c}\text { Difference } \\
\mathrm{dB}(\mathrm{A})\end{array}$ \\
\hline 1 & 40.3 & 50.2 & -9.9 \\
2 & 38.4 & 39.7 & -1.3 \\
3 & 38.7 & 38.7 & 0.0 \\
4 & 40.6 & 49.8 & -9.2 \\
\hline
\end{tabular}

As shown in Table 2, after adopting layout optimization, the noise values at the east and south boundaries reduced by $9.9 \mathrm{~dB}(\mathrm{~A})$ and $9.2 \mathrm{~dB}(\mathrm{~A})$ respectively. Due to the adjustment of the building location, the noise at the southeast boundary is increased with lower added value, and the increased noise value is less than $45 \mathrm{~dB}(\mathrm{~A})$, which meets the Class 1 noise standard at the boundary specified in GB12348.For the adjusted $220 \mathrm{kV}$ protection room, the manned room should be placed on the south side, so as to reduce the impact of transformer noise on staff.

\section{Conclusion}

In this paper, the method of substation layout optimization based on the maximum acoustic ray shielding method is established based on the sound wave propagation rule in a complicated air medium. Through the adjustment of the $220 \mathrm{kV}$ GIS room of a $220 \mathrm{kV}$ outdoor substation, the GIS room at southeast side is changed into a strip-shape room, and the strip-shaped building is moved to the south side of the main transformer, so as to control the noise at the south side of the boundary by shielding the maximum acoustic ray. Make full use of the feature that the height of the building is higher than the height of the sound sources to adjust the plane size of the original building, and increase the length width ratio of the building on the premise of meeting the functional use, so as to increase the area of the sound shadow zone behind the building. Finally, acoustic simulation analysis model of the $220 \mathrm{kV}$ outdoor substation is established, the noise level at the boundary of the substation before and after the layout optimization is compared and analyzed, and the distribution rule of the sound field inside and outside the substation is obtained. The analysis results show that the optimized layout of the substation can effectively reduce the noise at the boundary of the substation, which meets the limiting value requirements of Class 1 of the noise at the boundary of the substation. The layout optimization method of substation based on maximum acoustic ray shielding method provides efficient and feasible technical improvement measures for noise control at the boundary of substation.

\section{References}

1. $\mathrm{L} \mathrm{Yu}, \mathrm{J}$ Kang. Factors influencing the sound preference in urban open spaces. Applied Acoustics, 71 (2010).

2. HG Leventhall. Low frequency noise in buildings. Internal and external sources. Journal of Low Frequency Noise \&Vibration, 7 (1988).

3. K Persson-Waye, M Björkman. Annoyance due to low frequency noise and the use of the dBA scale. Journal of sound and Vibration, 127 (1988).

4. U Landstrom, P Lofstedt. Noise and annoyance in working environments. Environment International, 11 (1990).

5. RP Kendig. Sound intensity survey of the comerford HVDC converter station [J]. IEEE Trans. Power Deliv. 4 (1989).

6. W Kitagawa, Y Ishihara, $\mathrm{T}$ Todaka. Analysis of structural deformation and vibration of a transformer core by using magnetic property of magnetostriction. Electrical Engineering in Japan, 172 (2010).

7. Y Gao,K Muramatsu, MJ Hatim. Design of a reactor driven by inverter power supply to reduce the noise considering electromagnetism and magnetostriction. IEEE Transaction on Magnetics, 46 (2010). 
8. A. J. Moses , P. I. Anderson , T.Phophongviwat. Localized surface vibration and acoustic noise emitted from laboratory-scale transformer cores assembled from grain-oriented electrical steel. IEEE Transactions on Magnetics, 52(2016).

9. C. H. Hsu, S. L. Lee, C. C. Lin, et al. Reduction of vibration and sound-level for a single-phase power transformer with large capacity . IEEE Transactions on Magnetics,51 (2015).

10. L. H. Zhu, Q. X. Yang, R. G. Yan, etal. Numerical computation for a new way to reduce vibration and noise due to magnetostriction and magnetic forces of transformer noise. Journal of Applied Physics,113 (2013). 